\title{
Aspect Sentiment Classification with both Word-level and Clause-level Attention Networks
}

\author{
Jingjing Wang ${ }^{1}$, Jie $\mathbf{L i}^{3}$, Shoushan $\mathrm{Li}^{1, *}$, Yangyang Kang ${ }^{2}$, Min Zhang ${ }^{1}$, Luo Si ${ }^{2}$, Guodong Zhou ${ }^{1}$ \\ ${ }^{1}$ School of Computer Science and Technology, Soochow University, China \\ ${ }^{2}$ Alibaba Group, China \\ ${ }^{3}$ School of Computer Science and Engineering, Southeast University, China \\ ${ }^{1}$ djingwang@gmail.com, \{lishoushan, minzhang, gdzhou\}@ suda.edu.cn, \\ 3 jennyetjieli@gmail.com, ${ }^{2}$ \{yangyang.kangyy, luo.si $\} @$ alibaba-inc.com
}

\begin{abstract}
Aspect sentiment classification, a challenging task in sentiment analysis, has been attracting more and more attention in recent years. In this paper, we highlight the need for incorporating the importance degrees of both words and clauses inside a sentence and propose a hierarchical network with both wordlevel and clause-level attentions to aspect sentiment classification. Specifically, we first adopt sentence-level discourse segmentation to segment a sentence into several clauses. Then, we leverage multiple Bi-directional LSTM layers to encode all clauses and propose a word-level attention layer to capture the importance degrees of words in each clause. Third and finally, we leverage another Bidirectional LSTM layer to encode the output from the former layers and propose a clause-level attention layer to capture the importance degrees of all the clauses inside a sentence. Experimental results on the laptop and restaurant datasets from SemEval-2015 demonstrate the effectiveness of our proposed approach to aspect sentiment classification.
\end{abstract}

\section{Introduction}

The past decade has witnessed an exploding interest in sentiment analysis from natural language processing and data mining communities due to its inherent challenges and wide applications [Pang and Lee, 2007; Liu, 2012]. As a finegrained sentiment classification task, aspect sentiment classification aims to identify the sentiment polarity for a particular aspect. For example, the sentence "The price was too high but the food was delicious" would be assigned with negative polarity for aspect "price" while with positive polarity for aspect "food". Early studies typically employ traditional supervised learning algorithms which focus on designing a bag of features such as bag-of-words to train a classifier (e.g., Support Vector Machine, SVM) [Jiang et al., 2011; Pérez-Rosas et al., 2012].

\footnotetext{
${ }^{*}$ Corresponding author
}

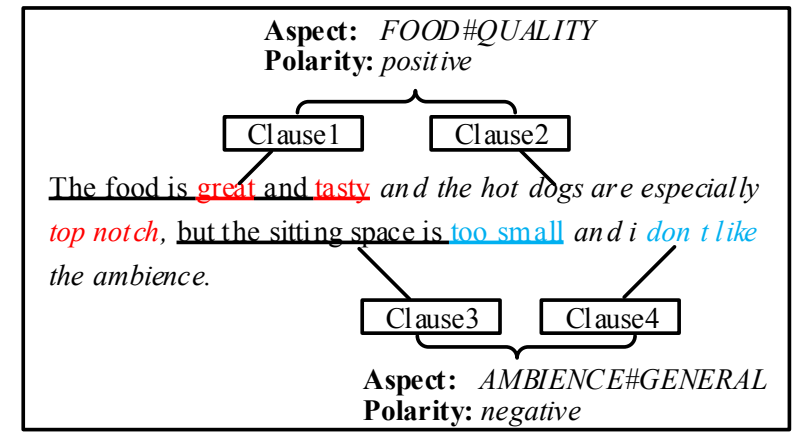

Figure 1: An example sentence in the restaurant domain where the entity $E$ and attribute $A$ pair (i.e., $E \# A$ ) defines the aspect category of the given text.

Recently, neural network approaches have shown promising results on sentiment classification, such as Recursive NN [Socher et al., 2011], Recursive NTN [Socher et al., 2013] and Tree-LSTM [Tai et al., 2015]. However, above neural network based approaches for sentiment classification just make use of the contexts without consideration of the aspect information while the aspect information should be an important factor for judging the aspect sentiment polarity. One possible way to incorporate the aspect information is to distinguish the importance of different texts with respect to a specific aspect.

First, for a specific aspect, the importance degrees of different words are different. For instance, in Figure 1, the words such as "great", "tasty" contribute much in implying the positive sentiment polarity for the aspect FOOD\#QUALITY. While, the words such as "is", "and" don't contribute. Therefore, a well-behaved neural network approach should consider the importance degrees of different words for predicting the sentiment polarity of a specific aspect.

Second, for a particular aspect, the importance degrees of different clauses are different. For instance, in Figure 1 , the first and second clauses have much stronger information in assisting the prediction of the sentiment polarity for the aspect FOOD\#QUALITY. In contrast, the third and fourth clauses are more relevant to the aspect $A M B I$ ENCE\#GENERAL. Therefore, a well-behaved neural net- 


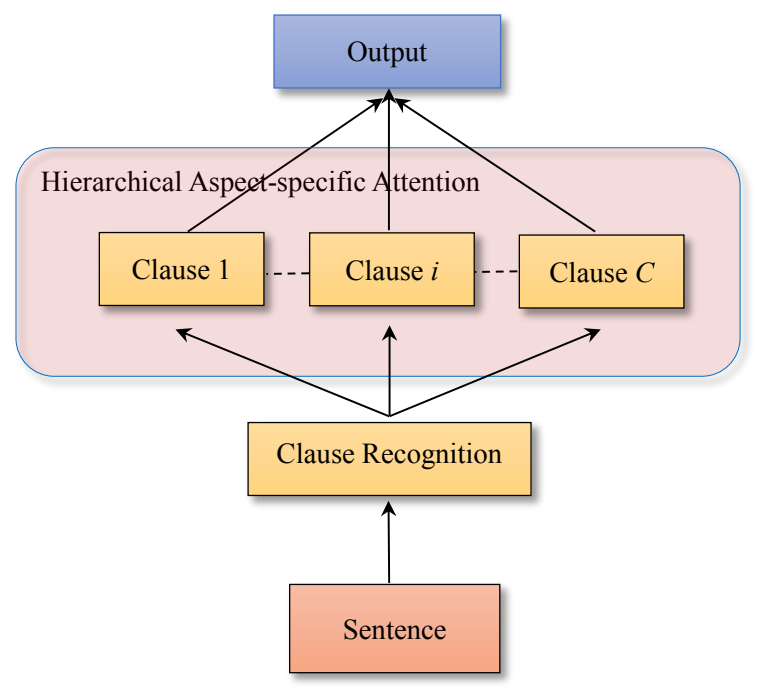

Figure 2: The overview of our approach

work approach should consider the importance degrees of different clauses for predicting the sentiment polarity of a specific aspect.

In particular, we propose a neural architecture, i.e., the Hierarchical Aspect-specific Attention Network, which leverages both word-level and clause-level attentions to incorporate the importance degrees of both words and clauses in a sentence. First, we adopt a sentence-level discourse segmentation method to segment a sentence into several clauses. Then, we leverage multiple Bi-directional LSTM layers to encode all clauses and propose a word-level attention layer to capture the importance degrees of words in each clause. Third, we leverage another Bi-directional LSTM to encode the output from the former Bi-directional LSTM layers and propose a clause-level attention layer to capture the importance degrees of all clauses. Experimental results on the laptop and restaurant datasets from SemEval-2015 [Pontiki et al., 2015] demonstrate that our proposed approach outperforms a number of competitive baselines and even significantly performs better than the best-performed system Sentiue in the shared task of SemEval-2015 [Saias, 2015].

\section{Related Work}

\subsection{Aspect Sentiment Classification}

In the literature, aspect sentiment classification is typically regarded as a text classification problem. Therefore, text classification approaches, such as SVM [Jiang et al., 2011], can be naturally applied to solve the aspect sentiment classification task without consideration of the mentioned aspect. Traditional machine learning approaches mainly focus on feature engineering to train a sentiment classifier [Jiang et al., 2011; Pérez-Rosas et al., 2012] and unable to discover the discriminative or explanatory factors of data. To solve this problem, [Dong et al., 2014] transfer a dependency tree of a sentence into a target-specific recursive structure, and get higher level representation based on that structure. [Vo and Zhang, 2015] use rich features including sentiment-specific word embedding and sentiment lexicons. [Guan et al., 2016] propose a novel deep learning framework for review sentiment classification which employs prevalently available ratings as weak supervision signals. [Tang et al., 2016b] propose a neural based approach that determines sentiment towards a target word based on its position.

\subsection{Aspect Sentiment Classification with Neural Networks}

Recently, neural network approaches have shown promising results on sentiment classification, such as Recursive NN [Socher et al., 2011], Recursive NTN [Socher et al., 2013] and Tree-LSTM [Tai et al., 2015]. However, the neural network based approaches just make use of the contexts without consideration of aspects which also make great contributions to judging the sentiment polarity of aspect.

Therefore, in order to incorporate aspects into a model, [Tang et al., 2016a] develop two long short-term memory (LSTM) to model the left and right contexts with target. [Wang et al., 2016] propose an attention-based LSTM in order to explore the potential correlation of aspects and sentiment polarities in aspect sentiment classification. [Tang et $a l ., 2016 \mathrm{~b}$ ] design deep memory networks which consist of multiple computational layers to integrate the target information. [Chen et al., 2017] also propose a deep memory network to integrate the target information, but the results of multiple attentions are non-linearly combined with a recurrent neural network. [Ma et al., 2017] propose an interactive learning approach, which interactively learns attentions in the contexts and targets.

Although above deep neural network models have achieved great success on aspect sentiment classification, they all ignore the incorporating knowledge of clause-level information in the model architecture. To the best of our knowledge, we are the first to address aspect sentiment classification with both word-level and clause-level attentions.

\section{Hierarchical Aspect-specific Attention Network}

In this section, we first introduce a clause recognition method to segment a sentence into several clauses. Then, we propose a hierarchical aspect-specific attention model which can concentrate on both the informative words and clauses corresponding to a given aspect in detail. Figure 2 shows the overview of the proposed approach to aspect sentiment classification.

Clause recognition is a non-trivial problem. Fortunately, in the literature, the clause recognition could be seen as a sub-problem of discourse segmentation which has been well-studied in the NLP community. Specifically, discourse segmentation is the task of breaking a given text into non-overlapping segments called elementary discourse units (EDUs) [Carlson et al., 2001]. Each EDU could be seen as a clause. In this study, we employ sentence-level discourse segmentation, which aims to segment a sentence into EDUs [Soricut and Marcu, 2003]. There exist several kinds of discourse theories and each of them has its own specificities in terms of segmentation guidelines and size of units. In this study, we adopt Rhetorical Structure Theory (RST) [MANN, 


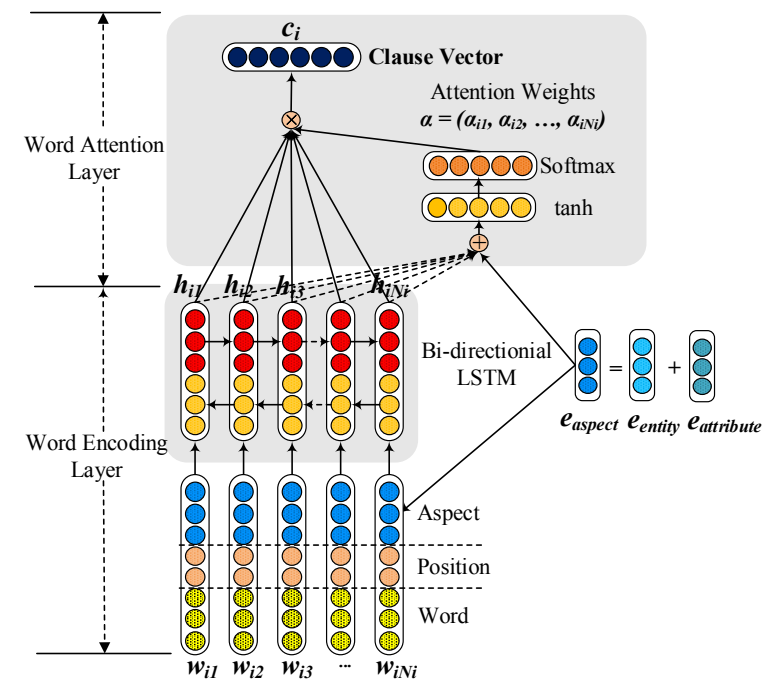

(a) Word-level Aspect-specific Attention Module

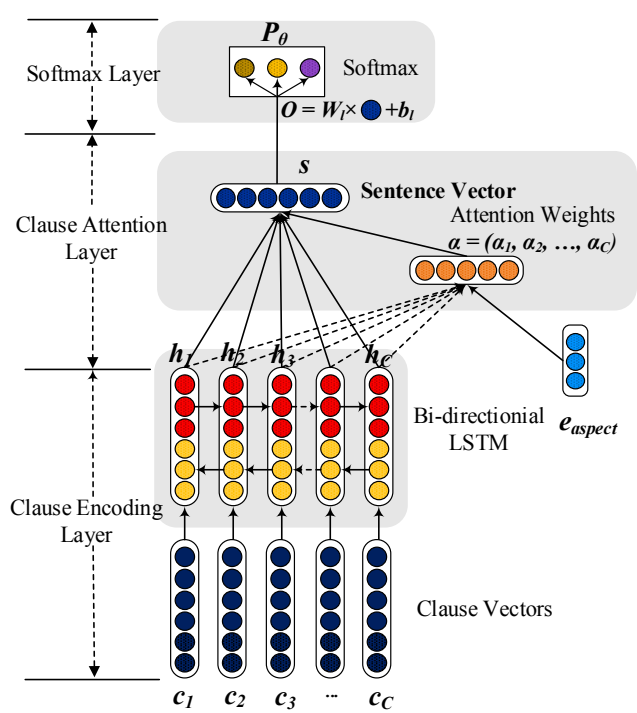

(b) Clause-level Aspect-specific Attention Module

Figure 3: The overall architecture of our proposed hierarchical aspect-specific attention approach

1988] due to its well-defined EDUs and perform sentencelevel discourse segmentation to detect EDUs as clauses. For instance, after the sentence-level discourse segmentation, the example in Figure 1 is segmented into four non-overlapping clauses, i.e., $1 A, 1 B, 1 C$ and $1 D$, as shown in E1.

E1: [The food is great and tasty] ${ }^{1 A}$ [and the hot dogs are especially top notch, ${ }^{1 B}$ [but the setting space is too

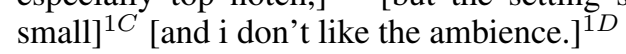

In the following, we introduce our hierarchical aspectspecific attention model to extract both the informative words and clauses corresponding to the specific aspect. Figure 3 shows the overall architecture of this approach which mainly consists of two components, i.e., a word-level aspect-specific attention module and a clause-level aspect-specific attention module. We will describe the details of the two modules as follows.

\subsection{Word-level Aspect-specific Attention}

Word Encoding Layer. Assume that a sentence has been segmented into $C$ clauses $c_{i}$ and each clause contains $N_{i}$ words. $I_{i j}$ represents the $j$-th word in the $i$-th clause. Given a clause $c_{i}$ with the word $I_{i j}$, the vector representation $w_{i j} \in$ $\mathbb{R}^{d=d_{w}+d_{p}}$ of word $I_{i j}$ consists of its word embedding and position embedding [Zeng et al., 2014], which is calculated as $w_{i j}=E_{w} \cdot I_{i j} \oplus E_{p} \cdot I_{i j}$ where $E_{w} \in \mathbb{R}^{d_{w} \times|V|}$ is word embedding matrix and $E_{p} \in \mathbb{R}^{d_{p} \times\left|V^{\prime}\right|}$ is position embedding matrix.

An aspect category consists of an entity and an attribute [Pontiki et al., 2015]. Specifically, the entity string $e_{\text {entity }}$ of length $L_{1}$ is represented as $\left\{x_{1}, \ldots, x_{L_{1}}\right\}$ where $x_{n} \in \mathbb{R}^{d^{\prime}}$ is the $d^{\prime}$-dimensional vector of the $n$-th word in the entity string. The attribute string $e_{\text {attribute }}$ is represented as $\left\{z_{1}, \ldots, z_{L_{2}}\right\}$. Since the common word embedding representations exhibit linear structure that makes it possible to meaningfully combine words by an element-wise addition of their vector representations, we use the sum of the entity and attribute embeddings to obtain a more compact aspect representation, i.e.,

$$
e_{\text {aspect }}=e_{\text {entity }}+e_{\text {attribute }}=\frac{1}{L_{1}} \sum_{n=1}^{L_{1}} x_{n}+\frac{1}{L_{2}} \sum_{n=1}^{L_{2}} z_{n}
$$

Then, inspired by [Tang et al., 2016a], we append the aspect representation to the embedding of each word to form an aspect-augmented embedding for each word $j$, i.e.,

$\hat{w}_{i j}=w_{i j} \oplus e_{\text {aspect }} ; \quad \hat{w}_{i j} \in \mathbb{R}^{d+d^{\prime}}, i \in[1, C], j \in\left[1, N_{i}\right]$

where $\oplus$ denotes the concatenate operator. $C$ is the number of clauses and $N_{i}$ is the number of words in the clause $c_{i}$. Noted that the dimension of $\hat{w}_{i j}$ is $\left(d+d^{\prime}\right)$.

Then, we use a Bi-directional LSTM (namely, Bi-LSTM) [Graves et al., 2013], which can efficiently make use of past features (via forward states) and future features (via backward states) for a specific time frame, to get annotations of words by summarizing information from both directions for words. The Bi-LSTM contains the forward LSTM $\vec{f}$ which reads the clause $c_{i}$ from the word $I_{i, 1}$ to $I_{i, N_{i}}$ and a backward LSTM $\overleftarrow{f}$ which reads from $I_{i, N_{i}}$ to $I_{i, 1}$ :

$$
\begin{aligned}
& \vec{h}_{i j}=\overrightarrow{\operatorname{LSTM}}\left(\hat{w}_{i j}\right) ; \quad i \in[1, C], j \in\left[1, N_{i}\right] \\
& \overleftarrow{h}_{i j}=\overleftarrow{\operatorname{LSTM}}\left(\hat{w}_{i j}\right) ; \quad i \in[1, C], j \in\left[N_{i}, 1\right]
\end{aligned}
$$

We obtain an annotation for a given word $I_{i j}$ by concatenating the forward hidden state $\vec{h}_{i j}$ and backward hidden state $\overleftarrow{h}_{i j}$ as follows:

$$
h_{i j}=\vec{h}_{i j} \oplus \overleftarrow{h}_{i j}
$$

which summarizes the information of the whole clause centered around the word $I_{i j}$. 
Word Attention Layer. Traditional LSTM model cannot capture the information about which words are important to the meaning of the clause. In order to address this problem, we design an attention mechanism which drives the model to concentrate on such words in the clause with respect to a specific aspect and aggregate the representation of those informative words to form a clause vector.

Figure 3(a) shows the details of the word-level attention module. Specifically, the following formulas are applied to compute the attention weight $\alpha_{i j}$ (similarity or relatedness) between each word annotation $h_{i j}$ and the aspect representation $e_{\text {aspect }}$.

$$
\begin{gathered}
u_{i j}=\tanh \left(W_{w} \cdot\left[h_{i j} ; e_{\text {aspect }}\right]+b_{w}\right) \\
\alpha_{i j}=\operatorname{softmax}\left(u_{i j}\right)=\frac{\exp \left(u_{i j}\right)}{\sum_{t=1}^{N} \exp \left(u_{i t}\right)}
\end{gathered}
$$

where $\left[h_{i j} ; e_{\text {aspect }}\right]$ denotes the vertical concatenation of $h_{i j}$ and $e_{\text {aspect }}, 1 \leq j \leq N_{i}, W_{w}$ is an intermediate matrix and $b_{w}$ is an offset value. $\alpha=\left[\alpha_{i 1}, \alpha_{i 2}, \ldots, \alpha_{i N_{i}}\right]$ are the weight vector of all words.

Then we compute the clause vector $c_{i}$ as a weighted sum of the word annotations based on the weights, i.e.,

$$
c_{i}=\sum_{i=1}^{N_{i}} \alpha_{i j} \cdot h_{i j}
$$

\subsection{Clause-level Aspect-specific Attention}

Clause Encoding Layer. Given the clause vectors $c_{i}$, we also use a Bi-LSTM to encode the clauses in order to incorporate the contextual information in the annotations, i.e.,

$$
\begin{aligned}
\vec{h}_{i}=\overleftrightarrow{\operatorname{LSTM}}\left(c_{i}\right) ; \quad i \in[1, C] \\
\overleftarrow{h}_{i}=\overleftarrow{\operatorname{LSTM}}\left(c_{i}\right) ; \quad i \in[C, 1]
\end{aligned}
$$

Similarly, we obtain an annotation for the clause $c_{i}$ by concatenating $\vec{h}_{i}$ and $\overleftarrow{h}_{i}$ as follows:

$$
h_{i}=\vec{h}_{i} \oplus \overleftarrow{h}_{i}
$$

which summarizes the information of the whole sentence centered around the clause $c_{i}$.

Clause Attention Layer. Figure 3(b) shows the details of the clause-level attention module. In this figure, $\left[h_{1}, h_{2}, \ldots, h_{C}\right]$ are annotation vectors for the clauses. With these context clause representations, we compute the attention weight $\alpha_{i}$ between each clause annotation $h_{i}$ and the aspect representation $e_{\text {aspect }}$ as follows:

$$
\begin{gathered}
m_{i}=\tanh \left(W_{c} \cdot\left[h_{i} ; e_{\text {aspect }}\right]+b_{c}\right) \\
\alpha_{i}=\operatorname{softmax}\left(m_{i}\right)=\frac{\exp \left(m_{i}\right)}{\sum_{t=1}^{C} \exp \left(m_{t}\right)}
\end{gathered}
$$

where $1 \leq i \leq C, W_{c}$ is an intermediate matrix, $b_{c}$ is an offset value. In addition, $e_{\text {aspect }}$ is the same as that in Equation (6), which is also calculated according to Equation (1).
After computing the clause annotation weights, we can get the sentence representation $s$ based on the attention vectors $\alpha_{i}$ by:

$$
s=\sum_{i=1}^{C} \alpha_{i} \cdot h_{i}
$$

Softmax Layer. To perform aspect sentiment classification, we feed the sentence representation $s$ to a softmax classifier, i.e.,

$$
o=W_{l} \cdot s+b_{l}
$$

where $o \in \mathbb{R}^{K}$ is the output, $W_{l}$ is the weight matrix and $b_{l}$ is the bias. Then, the probability of labeling sentence with sentiment polarity $k \in[1, K]$ is computed by:

$$
p_{\theta}=\frac{\exp \left(o_{k}\right)}{\sum_{t=1}^{K} \exp \left(o_{t}\right)}
$$

where $\theta$ denotes all parameters. Finally, the label with the highest probability stands for the predicted sentiment polarity of the aspect.

\subsection{Model Training}

We use cross-entropy loss function to train our model end-toend given a set of training data $x_{t}, e_{t}, y_{t}$, where $x_{t}$ is the $t$-th text to be predicted, $e_{t}$ is the corresponding aspect and $y_{t}$ is one-hot representation of the ground-truth sentiment polarity for aspect $e_{t}$ and text $x_{t}$. We represent this model as a blackbox function $\phi(x, e)$ whose output is a vector representing the probability of sentiment polarity. The goal of training is to minimize the loss function:

$$
J(\theta)=-\sum_{t=1}^{M} \sum_{k=1}^{K} y_{t}^{k} \cdot \log \phi\left(x_{t}, e_{t}\right)+\frac{l}{2}\|\theta\|_{2}^{2}
$$

where $M$ is the number of training samples; $K$ is the category number and $l$ is a $L_{2}$ regularization to bias parameters.

In the above equation, the model parameters are optimized by using Adagrad [Duchi et al., 2011]. All the matrix and vector parameters are initialized with uniform distribution in $\left[-\sqrt{6 /\left(r+c^{\prime}\right)}, \sqrt{6 /\left(r+c^{\prime}\right)}\right]$, where $r$ and $c^{\prime}$ are the numbers of rows and columns in the matrices [Glorot and Bengio, 2010]. The dropout strategy is used in the Bi-directional LSTM layer in order to avoid over-fitting.

\section{Experimentation}

\subsection{Experimental Settings}

- Data Settings: We conduct experiments on two datasets (i.e., one from the laptop domain and the other from the restaurant domain) from SemEval-2015 Task $12^{1}$ [Pontiki et $a l ., 2015]$ to validate the effectiveness of our approach. Each dataset consists of many customers reviews and each review contains a list of aspects and corresponding sentiment polarities, i.e., positive, neutral or negative. We also set aside $10 \%$ from the training set as the development data which is used to tune algorithm parameters.

\footnotetext{
${ }^{1}$ The detail introduction of this task is available at http://alt.qcri.org/semeval2015/task12/
} 
Proceedings of the Twenty-Seventh International Joint Conference on Artificial Intelligence (IJCAI-18)

\begin{tabular}{|l|c|c|}
\hline Model & $\begin{array}{c}\text { Restaurant } \\
\text { (Accuracy) }\end{array}$ & $\begin{array}{c}\text { Laptop } \\
\text { (Accuracy) }\end{array}$ \\
\hline \hline Majority & 0.537 & 0.570 \\
LSTM [Wang et al., 2016] & 0.735 & 0.734 \\
TC-LSTM [Tang et al., 2016a] & 0.747 & 0.745 \\
ATAE-LSTM [Wang et al., 2016] & 0.752 & 0.747 \\
RAM [Chen et al., 2017] & 0.767 & 0.759 \\
IAN [Ma et al., 2017] & 0.755 & 0.753 \\
\hline Sentiue [Saias, 2015] & 0.787 & 0.793 \\
\hline Hierarchical Bi-LSTM & 0.763 & 0.767 \\
Word-Level ATT & 0.789 & 0.785 \\
Clause-Level ATT & 0.783 & 0.779 \\
Word\&Clause-Level ATT & $\mathbf{0 . 8 0 9}$ & $\mathbf{0 . 8 1 6}$ \\
\hline
\end{tabular}

Table 1: Accuracy on aspect sentiment classification about both the restaurant and laptop domains

- Word Representations: (1) PTE: This is a word embedding resource built by ourselves with PTE which is a semisupervised representation learning tool proposed by [Tang et $a l ., 2015]^{2}$. This tool could leverage both labeled and unlabeled data to build a large-scale heterogeneous network and use the network to train the word vectors. In our implementation, on one hand, the labeled data is collected from Amazon by [McAuley et al., 2015]. Specifically, we pick the domains, i.e., Books, CDs, Clothing, Electronics, Restaurant and Health and each review is automatically assigned with a positive category if its rating score is 4 or 5 and a negative category if its rating score is 1 or 2 . On the other hand, the unlabeled data is the data from SemEval-2015 Task, as introduced above. The vocabulary size is about 1.2 million and the dimensionality of word vector is 300 . (2) Position Embeddings: Inspired by [Zeng et al., 2014], we use position embeddings to specify the aspect words, i.e., entity and attribute words (if available, in the sentence). The position embedding corresponds to the relative distance from current word to the aspect word. For instance, in Figure 1, the relative distance from the word "great" to the aspect word "food" is 3 . In our experiments, the relative distance is mapped to a vector of dimension 100 .

- EDU: We run EDU splitting with the Discourse Segmenter Tool $^{3}$ on all the datasets.

- Hyper-parameters: In our experiments, the word embedding and position embedding are optimized during training. All out-of-vocabulary words are initialized by sampling from the uniform distribution $U(-0.01,0.01)$. The dimensions of attention vectors and LSTM hidden states are set to be 300 . The other hyper-parameters are tuned according to the development data. Specifically, the initial learning rate is 0.1 . The regularization weight of the parameters is $10^{-5}$, and the dropout rate is set to 0.25 .

- Evaluation Metrics: The performance is evaluated using Accuracy and Macro-Fl $(F)$ which is calculated as $F=$ $\frac{2 P R}{P+R}$, where the overall precision $P$ and recall $R$ are averaged on the precision/recall scores of all categories.

\footnotetext{
${ }^{2}$ The word embedding resource is released at https://github.com/jjwangnlp/PTE2ASC

${ }^{3}$ http://alt.qcri.org/tools/discourse-parser/
}

\begin{tabular}{|l|c|c|}
\hline Model & $\begin{array}{c}\text { Restaurant } \\
(\text { Macro-F1 })\end{array}$ & $\begin{array}{c}\text { Laptop } \\
\text { (Macro-F1) }\end{array}$ \\
\hline \hline Majority & 0.233 & 0.242 \\
LSTM [Wang et al., 2016] & 0.617 & 0.608 \\
TC-LSTM [Tang et al., 2016a] & 0.634 & 0.622 \\
ATAE-LSTM [Wang et al., 2016] & 0.641 & 0.637 \\
RAM [Chen et al., 2017] & 0.645 & 0.639 \\
IAN [Ma et al., 2017] & 0.639 & 0.625 \\
\hline Sentiue [Saias, 2015] & 0.660 & 0.634 \\
\hline Hierarchical Bi-LSTM & 0.647 & 0.632 \\
Word-Level ATT & 0.662 & 0.646 \\
Clause-Level ATT & 0.659 & 0.647 \\
Word\&Clause-Level ATT & $\mathbf{0 . 6 8 5}$ & $\mathbf{0 . 6 6 7}$ \\
\hline
\end{tabular}

Table 2: Macro-F1 on aspect sentiment classification about both the restaurant and laptop domains

\subsection{Experimental Results}

In this subsection, we give some baseline approaches for comparison in order to comprehensively evaluate the performance of our proposed approach. Note that all these learning approaches employ the same representations, i.e., word PTE embedding together with the position embedding.

- Majority: This approach is a basic baseline approach, which assigns the majority sentiment polarity in the training set to each sample in the test set.

- LSTM: This approach only uses one LSTM network to model the context. After that, the average value of all the hidden states is fed to a softmax function to estimate the probability of each sentiment label [Wang et al., 2016].

- TC-LSTM: This approach extends LSTM by taking into account of the aspect information where two LSTM networks, a forward one and backward one towards the aspect, are adopted. This is a state-of-the-art approach to aspect sentiment classification proposed by [Tang et al., 2016a].

- ATAE-LSTM: This approach models the context words via attention-based LSTM and appends the aspect embeddings with each word embedding vector. This is a state-ofthe-art approach proposed by [Wang et al., 2016].

- RAM: This approach captures importance of context words for a specific aspect with a deep memory network and the results of multiple attentions are non-linearly combined with a recurrent neural network. This is a state-of-the-art approach proposed by [Chen et al., 2017].

- IAN: This approach is an interactive learning approach, which firstly models the contexts and aspects via LSTM and then interactively learns attentions in the contexts and aspects. This is another state-of-the-art approach proposed by [Ma $e t$ al., 2017].

- Sentiue: This is the best-performed system in SemEval2015 Task 12 [Saias, 2015]. It employs a MaxEnt classifier with n-gram features, POS features, lexicon features and achieves the best accuracy scores in both the domains laptop and restaurant.

- Hierarchical Bi-LSTM: Our approach which employs neither the word-level nor clause-level attention. 


\section{Aspect: FOOD\#QUALITY}

Polarity: positive

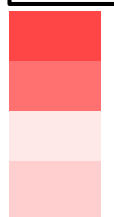

the food is great and tasty
and the hot dogs are especially top notch,
but the sitting space is too small
and ido n't like the ambience.

(a) the aspect of this sentence : FOOD\#QUALITY

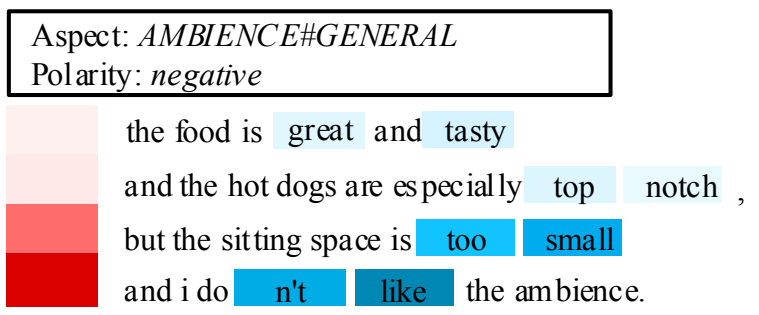

(b) the aspect of this sentence : AMBIENCE\#GENERAL

Figure 4: The attention visualizations for a sentence including two different aspects, i.e., "FOOD\#QUALITY" and "AMBIENCE\#GENERAL"

- Word-Level ATT: Our approach which employs only the word-level attention.

- Clause-Level ATT: Our approach which employs only the clause-level attention.

- Word\&Clause-Level ATT: Our approach which employs both the word-level and clause-level attentions.

Table 1 and Table 2 show the performance comparison of different approaches. From these two tables, we can see that, all LSTM-based models perform better than the Majority approach, showing that LSTM has potentials in automatically generating representations and can all bring performance improvement for sentiment classification.

The four state-of-the-art approaches, i.e., TC-LSTM, ATAE-LSTM, RAM and IAN, all perform better than LSTM. These results confirm the helpfulness of considering aspect information in aspect sentiment classification. Moreover, we find that ATAE-LSTM, RAM and IAN perform a bit better than TC-LSTM, which demonstrates that using attention mechanism is a good choice to model aspect information.

Our approach Hierarchical Bi-LSTM outperforms most of the state-of-the-art approaches, which highlights the importance of employing clause information in a sentence. When the word-level or clause-level attention is leveraged, our approach Word-Level ATT and Clause-Level ATT achieve better performance and outperform all the state-ofthe-art approaches. Among all these approaches, our approach Word\&Clause-Level ATT performs best and even outperforms the top-performed system of SemEval-2015 Task 12, i.e., Sentiue. Impressively, compared to LSTM, Word\&Clause-Level ATT achieves the average improvement of $7.4 \%$ (Accuracy), 6.8\% (Macro-F1) on the restaurant dataset and $8.2 \%$ (Accuracy), 5.9\% (Macro-F1) on the laptop dataset. These results encourage to incorporate the importance degrees of both words and clauses in a sentence.

\subsection{Discussion: Visualization of Attention}

In order to get a better understanding of our hierarchical aspect-specific attention model and validate that this model is able to select informative words and clauses corresponding to a specific aspect in a sentence, we visualize the word-level attention layers and clause-level attention layers according to the obtained attention weight $\alpha$ in Equation (7) and Equation (13) respectively.

Figure 4 shows the attention visualizations for a sentence including two different aspects, i.e., FOOD\#QUALITY and $A M B I E N C E \# G E N E R A L$, from the restaurant dataset. Here, we use the visualization approach proposed by [Yang et al., 2016]. Specifically, we normalize the word weight by the clause weight to make sure that only informative words in informative clauses corresponding to a given specific aspect are emphasized. In Figure 4, each line is a clause. Red denotes the clause weight and blue denotes the word weight. The color depth indicates the importance degree of attention weight for a specific aspect, the darker the more important.

From this figure, we can see that the clause-level attention function always selects the informative clauses corresponding to a specific aspect, such as selecting the first and second clauses for the aspect FOOD\#QUALITY; while selecting the third and fourth clauses for the aspect $A M B I$ ENCE\#GENERAL. In addition, the word-level attention function can select both the words and multi-word phrases carrying strong sentiment signals corresponding to a given specific aspect, such as "great", "tasty", "top notch" if the given aspect is FOOD\#QUALITY; while "too small" and " $n$ 't like" if the given aspect is AMBIENCE\#GENERAL.

\section{Conclusion}

In this paper, we propose a hierarchical aspect-specific attention model for aspect sentiment classification. The main idea of the proposed model is to segment a sentence into several clauses and then use both word-level and clause-level attentions to incorporate the knowledge of word-level and clauselevel text information. Experimental results on the laptop and restaurant datasets from SemEval-2015 demonstrate that the proposed approach outperforms a number of competitive baselines and even the best-performed system in the shared task of SemEval-2015.

In our future work, we would like to employ more information in the discourse analysis, e.g., relationships between two clauses, to improve the performance. Furthermore, we would like to apply our word-level and clause-level model to other sentiment analysis tasks, e.g., sentence-level sentiment classification.

\section{Acknowledgments}

The research work is partially supported by the National Key R\&D Program of China under Grant No.2017YFB1002101 and two NSFC grants No.61331011, No.61672366. This work is also supported by the joint research project of Alibaba and Soochow University. 


\section{References}

[Carlson et al., 2001] Lynn Carlson, Daniel Marcu, and Mary Ellen Okurovsky. Building a discourse-tagged corpus in the framework of rhetorical structure theory. In Proceedings of SIGDIAL-2001, 2001.

[Chen et al., 2017] Peng Chen, Zhongqian Sun, Lidong Bing, and Wei Yang. Recurrent attention network on memory for aspect sentiment analysis. In Proceedings of EMNLP-2017, pages 452-461, 2017.

[Dong et al., 2014] Li Dong, Furu Wei, Chuanqi Tan, Duyu Tang, Ming Zhou, and $\mathrm{Ke} \mathrm{Xu}$. Adaptive recursive neural network for target-dependent twitter sentiment classification. In Proceedings of ACL-2014, pages 49-54, 2014.

[Duchi et al., 2011] John Duchi, Elad Hazan, and Yoram Singer. Adaptive subgradient methods for online learning and stochastic optimization. Journal of Machine Learning Research, 12:2121-2159, 2011.

[Glorot and Bengio, 2010] Xavier Glorot and Yoshua Bengio. Understanding the difficulty of training deep feedforward neural networks. In Proceedings of AISTATS-2010, pages 249-256, 2010.

[Graves et al., 2013] Alex Graves, Abdel Rahman Mohamed, and Geoffrey Hinton. Speech recognition with deep recurrent neural networks. 38(2003):6645-6649, 2013.

[Guan et al., 2016] Ziyu Guan, Long Chen, Wei Zhao, Shulong Tan, Shulong Tan, and Deng Cai. Weakly-supervised deep learning for customer review sentiment classification. In Proceedings of IJCAI-2016, pages 3719-3725, 2016.

[Jiang et al., 2011] Long Jiang, Mo Yu, Ming Zhou, Xiaohua Liu, and Tiejun Zhao. Target-dependent twitter sentiment classification. In Proceedings of ACL-2011, pages 151160, 2011.

[Liu, 2012] Bing Liu. Sentiment Analysis and Opinion Mining. Synthesis Lectures on Human Language Technologies. Morgan \& Claypool Publishers, 2012.

[Ma et al., 2017] Dehong Ma, Sujian Li, Xiaodong Zhang, and Houfeng Wang. Interactive attention networks for aspect-level sentiment classification. In Proceedings of IJCAI-2017, pages 4068-4074, 2017.

[MANN, 1988] W. MANN. Rhetorical structure theory : Toward a functional theory of text organization. Text, 8(3):243-281, 1988.

[McAuley et al., 2015] Julian J. McAuley, Rahul Pandey, and Jure Leskovec. Inferring networks of substitutable and complementary products. In Proceedings of SIGKDD2015, pages 785-794, 2015.

[Pang and Lee, 2007] Bo Pang and Lillian Lee. Opinion mining and sentiment analysis. Foundations and Trends in Information Retrieval, 2(1-2):1-135, 2007.

[Pérez-Rosas et al., 2012] Verónica Pérez-Rosas, Carmen Banea, and Rada Mihalcea. Learning sentiment lexicons in spanish. In Proceedings of LREC-2012, pages 3077-3081, 2012.
[Pontiki et al., 2015] Maria Pontiki, Dimitris Galanis, Haris Papageorgiou, Suresh Manandhar, and Ion Androutsopoulos. Semeval-2015 task 12: Aspect based sentiment analysis. In Proceedings of NAACL-HLT-2015, pages 486-495, 2015.

[Saias, 2015] José Saias. Sentiue: Target and aspect based sentiment analysis in semeval-2015 task 12. In Proceedings of NAACL-HLT-2015, pages 767-771, 2015.

[Socher et al., 2011] Richard Socher, Jeffrey Pennington, Eric H. Huang, Andrew Y. Ng, and Christopher D. Manning. Semi-supervised recursive autoencoders for predicting sentiment distributions. In Proceedings of EMNLP2011, pages 151-161, 2011.

[Socher et al., 2013] R. Socher, A. Perelygin, J. Y. Wu, J. Chuang, C. D. Manning, A. Y. Ng, and C. Potts. Recursive deep models for semantic compositionality over a sentiment treebank. 2013.

[Soricut and Marcu, 2003] Radu Soricut and Daniel Marcu. Sentence level discourse parsing using syntactic and lexical information. In Proceedings of NAACL-2003, 2003.

[Tai et al., 2015] Kai Sheng Tai, Richard Socher, and Christopher D. Manning. Improved semantic representations from tree-structured long short-term memory networks. In Proceedings of ACL-2015, pages 1556-1566, 2015.

[Tang et al., 2015] Jian Tang, Meng Qu, and Qiaozhu Mei. PTE: predictive text embedding through large-scale heterogeneous text networks. In Proceedings of SIGKDD2015, pages 1165-1174, 2015.

[Tang et al., 2016a] Duyu Tang, Bing Qin, Xiaocheng Feng, and Ting Liu. Effective lstms for target-dependent sentiment classification. In Proceedings of COLING-2016, pages 3298-3307, 2016a.

[Tang et al., 2016b] Duyu Tang, Bing Qin, and Ting Liu. Aspect level sentiment classification with deep memory network. In Proceedings of EMNLP-2016, pages 214-224, $2016 b$.

[Vo and Zhang, 2015] Duy-Tin Vo and Yue Zhang. Targetdependent twitter sentiment classification with rich automatic features. In Proceedings of IJCAI-2015, pages 1347-1353, 2015.

[Wang et al., 2016] Yequan Wang, Minlie Huang, Xiaoyan Zhu, and Li Zhao. Attention-based LSTM for aspect-level sentiment classification. In Proceedings of EMNLP-2016, pages 606-615, 2016.

[Yang et al., 2016] Zichao Yang, Diyi Yang, Chris Dyer, Xiaodong He, Alexander J. Smola, and Eduard H. Hovy. Hierarchical attention networks for document classification. In Proceedings of NAACL-HLT-2016, pages 1480-1489, 2016.

[Zeng et al., 2014] Daojian Zeng, Kang Liu, Siwei Lai, Guangyou Zhou, and Jun Zhao. Relation classification via convolutional deep neural network. In Proceedings of COLING-2014, pages 2335-2344, 2014. 